\title{
Blockchain Technology in Healthcare
}

\author{
Serik Smagulov, \\ Viktoriya Smagulova MD
}

\begin{abstract}
This article discusses the possibility and validity of the use of blockchain in health care. When it comes to managing public health the biggest problems that have so far been encountered are data security, sharing and compatibility. If patient information is isolated and stored in several systems that do not allow for the smooth exchange of information, the population health data sets for different patient sets will be insufficient. Blockchain, according to the author, provides a reliable solution to this particular problem. The article discusses in detail the three basic principles of the blockchain: cryptography, distributed books and authentication. The distributed blockchain technology with such properties as immutability, reliability and decentralization, provides the healthcare sector with opportunities to detect fraud, reduce operating costs, smooth processes, eliminate duplication of work, and apply transparency in the health ecosystem. If used correctly, the blockchain will improve security, data exchange, compatibility, data integrity and update, and real-time access. The author of this article points out the importance of using the blockchain for data security in clinical trials, which always require a huge amount of data sets.
\end{abstract}

Keywords: blockchain, cryptography, distributed books, authentication, data, security.

In the health sector, critical patient data and information remain scattered across different departments and systems. Because of this, important data is not available and easily accessible if necessary.

The misuse of available data prevents health organizations from providing appropriate patient care and high-quality services to improve health. Even though these organizations are quite useful from an economic point of view, they cannot meet the needs of patients.

Many medical institutions today still rely on outdated patient record keeping systems. These systems support the functionality of maintaining local patient data records. This can make it difficult for the doctor to diagnose, which is time-consuming and tedious for patients. Due to this, the cost of a patient-centered work increases significantly.

Another time-consuming and tedious process that leads to high costs in health care is the exchange of medical information. Since patients have no control over their data, the likelihood of identity theft, financial data offenses, and spam increases every day. 
Compatibility is a huge problem in the healthcare industry [1]. Well-communicating systems can improve operational efficiency, reducing time spent on administrative tasks like manually entering data received from faxes [2]. Among the main problems, there are the problem of identifying patients and the problem of blocking information.

In this regard, Blockchain is a solution for many of the problems listed here.

Blockchain technology is one of the most important and destructive technology in the world. Many industries are adopting blockchain technology for innovation in their work. One of the sectors that are seeking to take the blockchain is the healthcare industry [3].

In the simplest sense, the blockchain is a series of permanent data records with timestamps that are managed by a cluster of computers that do not belong to any single entity. Each of these data blocks is protected and linked to each other using cryptographic principles.

The reason why the blockchain has become so popular is that it does not belong to one person; therefore, it is decentralized [4]. Also, data is cryptographically stored inside. The blockchain is immutable, so no one can interfere with the data that is inside the blockchain. The blockchain is transparent so that you can track data.

The work of the blockchain is based on three basic principles that have existed for a long time. The compiled work of these principles allows the blockchain to provide safe and secure digital relationships.

- Cryptography with a private key. In cryptography with a private key, the secret key is used as a variable along with the encryption and decryption algorithm. The key is kept secret, even if the algorithm is not. In the blockchain, a link to secure digital identification is created, but transactions are carried out in an open network.

- Distributed books. A distributed register, also known as a general register, is called a consensus of general records. In the DLT, the ledger is updated in real time, and no central authority is responsible for its maintenance. Instead, network members update the register. Any changes made to the ledgers are reflected within a few seconds. 
- Authentication. Authentication is a process that proves authenticity. In the blockchain, all transactions are authenticated before being added to the chain. This process takes place with the help of algorithms that check and verify all transactions. Once the information is encrypted, digitally signed and saved, the authenticity is sealed.

Blockchain can also help create a patient information exchange market $[5 ; 6]$. Thus, it will be possible to stimulate the exchange of information between different institutions to prevent any blocking information.

Because the blockchain is immutable and traceable, patients can easily send entries to anyone without fear of data corruption or tampering.

Similarly, the medical card that was generated and added to the blockchain will be fully protected.

A patient may have some control over how his medical records are used and transmitted by the institutes. Any party who wants to obtain medical data about a patient can check with the blockchain to obtain the necessary permission.

Various medical institutions around the world conduct their research and clinical trials of multiple new drugs and medicines. The blockchain will help create a single global database to collect all this data and put it in one place.

According to a study conducted by IBM, about $16 \%$ of health managers have decided on their plans to implement the blockchain solution in their work this year, while about 56\% expect to perform the blockchain by 2020 .

Blockchain allows you to create and distribute a single common health database.

This system will be available to all persons involved in the process, regardless of which electronic medical system they use. This provides a higher level of safety and transparency, allowing physicians to devote more time to patient care and treatment. Also, it will also allow for a better exchange of research statistics, which, in turn, will facilitate clinical trials and treatment of diseases [7] 
Participants can share and track their data and other activities taking place in the system without having to search for additional options to ensure integrity and security.

In accordance with the requirements and access permissions for network participants, two types of block chains can be used: allowed blockchains and block chains without access rights, which would enable anyone to create their address and start interacting with the network.

The distributed blockchain technology, with properties such as immutability, reliability, and decentralization, provides the healthcare sector with opportunities to detect fraud, reduce operating costs, smooth processes, eliminate duplication of work, and apply transparency in the health ecosystem [8].

The existing health care system and organizations work through a single central database. A single entity manages this database in the organization. With this approach, the point of failure is also reduced to a single point.

In such cases, if a hacker or an antisocial element attacks the system, a person can access the entire database and put at risk both the patient and the organization [9].

Blockchain can be used to prevent an organization's internal infrastructure. A large organization with several independent entities with different levels of access in the register of blockchains with encryption built into the blocks will save organizations from external threats and attacks.

If a blockchain network is correctly implemented in a health organization, this will prevent such redemption attacks, as well as other problems, such as data corruption or equipment failure.

Blockchain can also be used for data security in clinical trials. Therefore, a vast number of data sets are required for a clinical trial. Researchers focus on these datasets and conduct regular tests under various circumstances to generate reports, statistics, and performance ratios [10]. 
Based on these reports, data is analyzed, and further decisions are made. Research documents can be stored as smart contracts on the blockchain, acting as digital fingerprints.

With regard to the security of personal data, the blockchain system for managing patient data can create a hash for individual blocks of patient health information, and the collective system will be a patient identifier on a theoretical basis.

The blockchain system will allow patients to disclose the necessary data to third parties while maintaining their identity in secret. Patients can also control the timing and access rights for exchanging data with third parties.

As we see, the use of blockchain in health care is more than justified [11]. The only problem now is to force medical workers to implement blockchain systems on a larger scale.

\section{References}

1. William J. Gordon, Christian Catalini. Blockchain Technology for Healthcare: Facilitating the Transition to Patient-Driven Interoperability // Comput Struct Biotechnol J. 2018; 16: 224-230. Published online 2018 Jun 30.

2. Zhou Y., Ancker J.S., Upadhye M., McGeorge N.M., Guarrera T.K., Hegde S. The impact of interoperability of electronic health records on ambulatory physician practices: A discrete-event simulation study. Inform Prim Care. 2013;21:21-29.

3. Mettler M. Blockchain technology in healthcare: The revolution starts here. 2016 IEEE 18th Int. Conf. E-Health Netw. Appl Serv Heal. 2016:1-3.

4. Gordon W.J., Wright A., Landman A. Blockchain Technology in Health Care: Decoding the hype. NEJM Catal. 2017 https://catalyst.nejm.org/decodingblockchain-technology-health/

5. Dubovitskaya A, Xu Z, Ryu S, Schumacher M, Wang F. Secure and trustable electronic medical records sharing using blockchain. AMIA Annu Symp Proc 2017;2017:650-659

6. Meinert E, Alturkistani A, Foley KA, Osama T, Car J, Majeed A, et al. Blockchain Implementation in Health Care: Protocol for a Systematic Review. JMIR Res Protoc 2019 Feb 08;8(2):e10994 
7. Liang X, Zhao J, Shetty S, Liu J, Li D. Integrating Blockchain for Data Sharing and Collaboration in Mobile Healthcare Applications. 2017 Oct 8 Presented at: 2017 IEEE 28th Annual International Symposium on Personal, Indoo

8. Zyskind Guy, Nathan Oz, Pentland Alex. Decentralizing privacy: Using blockchain to protect personal data. IEEE Secur Priv Work. 2015;2015:180-184.

9. Walker J., Pan E., Johnston D., Adler-Milstein J., Bates D.W., Middleton B. The value of health care information exchange and interoperability. Health Aff Proj Hope. 2005 doi: 10.1377/hlthaff.w5.10. Suppl Web Exclusives: W5-10-W5-18

10. Liam Bell, William J Buchanan, Jonathan Cameron, Owen Lo. Applications of Blockchain Within Healthcare // Blockchain in Healthcare Today, 08 Jul 2018. DOI: $10.30953 /$ bhty.v1.8

11. Diane J. Skiba. The Potential of Blockchain in Education and Health Care // Nursing Education Perspectives: July/August 2017 - Volume 38 - Issue 4 - p 220-221 